KLEINE TEXTE FÜR THEOLOGISCHE UND PHILOLOGISCHE VORLESUNGEN UND ÜBUNGEN HERAUSGEGEBEN VON HANS LIETZMANN

\title{
LITURGISCHE TEXTE
}

V

\section{MARTIN LUTHERS}

DEUTSCHE MESSE

I 526

HERAUSGEGEBEN

VON

D. HANS LIETZMANN

O. PROFESSOR IN JENA

PREIS: $0,40 \mathrm{M}$.

BONN

A. MARCUS UND E. WEBER'S VERLAG 
H. Gressmann, W. Eeitmüller, I. Klostermann, B. Preuschen, I. Radermacher, H. Schlosser,

F. Niebergall, H. Windisch.

herausgegeben von

\section{Hans Lietzmann}

Erschienen sind:

Erste Iieferung: (Band III. Die Briefe des Apostels Paulus Bogen I-5.) An die Römer. Erklärt von Hans Lietzmann. Subskr.-Preis M. 1.35. Einzelpreis M. 1.50.

Zweite Iieferung. (Band V I, Bogen $\mathrm{I}-3$ und $\mathrm{V}_{2}$ Bogen I-3.) Allgemeine Finleitung zux praktischen Auslegung nnd praktische Auslegung zum Römerbrief. Ton $F$. Niebergall. Subskr.-Preis M. I.60. Einzelpreis M. I.80.

Dritte und vierte Iieferung. (Band I 2.) Die hellenistischrömische Kultur in ihren Beziehungen zu Judentum and Christentum. Von P. Wendland. Mit 5 Abbild. u. 12 Tafln. Subskr.-Preis M. 4.50. Einzelpreis M. 5.-. Gebunden M. 7.-.

Fünfte Iieferung. (Band III. Die Briefe des Apostels Paulus Bogen 6-1 1.) An die Corinther I. Erklärt von Hans Liet $z$. mann. Subskr.-Preis M. I.44. Einzelpreis M. I.6o.

Sechste (Doppel-) Iieferung. (Band II. Die Fvangelien Bogen I--Io.) Markus. Unt. Mitwkg. von Hugo Gressmann erklärt v. Erich Klostermann. Subskr.-Preis M. 2.56. Einzelpr. M. 2.85.

Siebente Iieferung. (Bd. V I, Bg. 4-8.) Praltische Auslegung zu Markus. Von Fr. Ni be rgall. Sub.-Pr. N. r.26. Ezpr. M. I.40.

Achte Lieferung: (Band V I, Bogen 9 und ro und V 2, Bogen 4 und 5.) Praktische Auslegung zu Matthäug und zu I. Cor.

Von Fr. Niebergall. Subskr.-Preis M. I.08. Einzelpreis M. 1.20.

Neunte Iieferung. (Bd. V I, Bogen I I-I4.) Praktische Auslegung au Iukas und Apostelgeschichte. Von Fr. Niebergall. Subskr.-Preis M. 1.08. Einzelpreis M. I.20.

Zehnte Inieferung. (Bd. V I, Bg. I5-I 8.) Praktische Auslegung za Johannes. V. Fr. Nie berg a 1l. Sub.-Pr. M. 1.08. Ez.-Pr. M. T.2O:

Die in sich abgeschlossenen Lieferungen und Bände des Werkes werden einzeln abgegeben.

Prospekte und Textproben stehen zur Verfügung.

\section{Ubersicht thber das Sammelwerk:}

Band I: I. Grammatik des neutestamentlichen Griechisch von L. Rader * macher. 2. Die hellenistisch-römische Kultur in ihren Beziehungen zu Judentum und Christentum von P. Wendland. (Erschienen. Einzelpreis M. 5.-. Gebd. M. 7.-) 3. Die urchristlichen Literaturformen von $P$. $\mathrm{W}^{r}$ endland.

Band II: I. Die Synoptiker unter Mitwirkung von FI. Gressmann, erklärt von E. Klostermann. Lirschicnen Markus: Lieferung 6 . 2. Johannesevangelium, erklärt von $\mathrm{E}$. Klostermann.

Band III: I. Die vier paulinischen Hauptbriefe erklärt von H. L i etz mann. Erschienen Römerbrief und I. Korintherbrief: Lfg. I u. 5 . 2. Die neun übrigen paulinischen Briefe erklärt von $E$. Klostermann und H. Liet $z$ mann.

(Fortsetzung siehe 3. Umschlagseite.) 